cystic carcinoma was occult on MRI possibly obscured by intense parenchymal background enhancement. Our histopathological findings of invasive adenoid cystic carcinoma confirm the importance of pathological examination of reduction mammoplasty specimens for breast carcinoma screening. It is also clear that a more extensive evaluation should be performed in patients with a history of breast carcinoma. Adequate sampling of reduction specimens is essential for accurate diagnosis and is vital for optimal clinical management.

\title{
Alveolar Variant of Invasive Lobular Carcinoma in a Fibroadenoma
}

\author{
Randall Butler, MD,* Renee Pinsky, MD, ${ }^{\dagger}$ and Julie M. Jorns, MD* \\ *Department of Pathology, University of Michigan, Michigan; and Department of Radiology, Univer- \\ sity of Michigan, Michigan
}

A 46-year-old female had a breast mass noted on routine screening mammogram [Fig. 1]. Ultrasound confirmed the mass to be circumscribed, oval, and hypoechoic, measuring $0.8 \times 0.8 \times 0.6 \mathrm{~cm}$. Imaging was largely unchanged (from up to 5 years prior) and the lesion favored to be benign.

Biopsy revealed an epithelial proliferation with lobular expansion, pagetoid extension into ducts, and back-to-back well-circumscribed nests comprised of small cells with low mitotic rate and classic lobular histology characterized by discohesion, intracytoplasmic lumina, and signet ring forms. Immunohistochemical stains highlighted myoepithelial cells surrounding in situ carcinoma cells and a lack of myoepithelial staining in the back-to-back nests, confirming invasive carcinoma. E-cadherin showed a lack of staining in neoplastic cells, confirming the lobular nature and morphological impression of invasive lobular carcinoma (ILC), alveolar variant, with associated lobular carcinoma in situ (LCIS) [Fig. 2]. The subsequent wire-localization lumpectomy specimen was remarkable for a $1.0 \mathrm{~cm}$ well-circumscribed, gray-white, solid mass with homogenous cut surfaces. The mass had a lobulated architecture with compressed ducts and background spindled-to sclerotic stroma charac-

Address correspondence and reprint requests to: Julie M. Jorns, MD, University of Michigan, Department of Pathology, 1500 East Medical Center Drive 2G332 UH, Ann Arbor, Michigan 48109, USA, or e-mail: jjorns@med. umich.edu.

DOI: $10.1111 /$ tbj.12016

(C) 2012 Wiley Periodicals, Inc., 1075-122X/12

The Breast Journal, Volume 18 Number 6, 2012 613-614

teristic of intracanalicular fibroadenoma (FA). As seen previously, the FA was largely replaced by ILC, alveolar variant, modified Bloom-Richardson grade 1, and multifocal LCIS [Fig. 3]. Surrounding breast parenchyma was unremarkable.

Individually, both the alveolar variant of ILC and carcinoma limited to a FA are uncommon presentations of breast cancer. Although the alveolar variant mimics LCIS, tumor nests are subtly infiltrative,

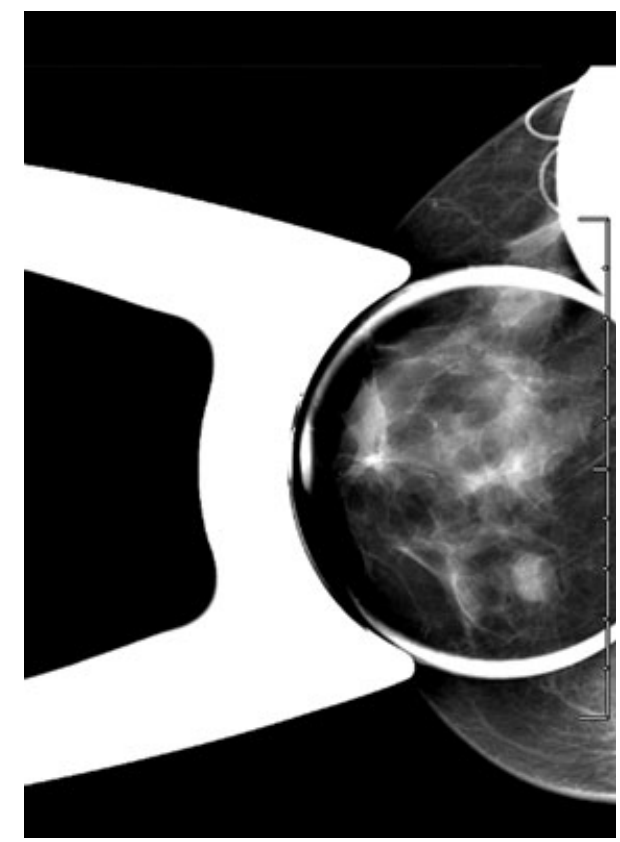

Figure 1. Diagnostic digital spot compression craniocaudal mammogram view of the mass demonstrating the oval shape with circumscribed margins. 

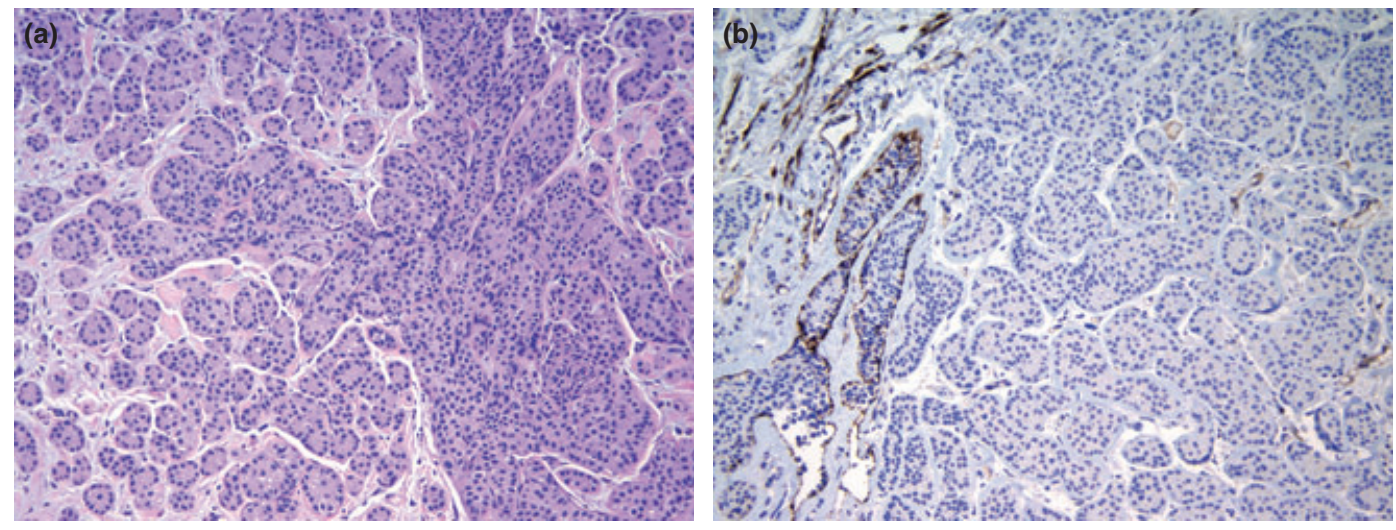

Figure 2. Needle core biopsy with epithelial expansion of lobules, pagetoid extension into ducts, and infiltrative, back-to-back nests exhibiting lobular cytology (a). Myoepithelial marker muscle-specific actin (MSA) demonstrating lack of staining in invasive carcinoma and appropriate myoepithelial staining in carcinoma in situ (b).

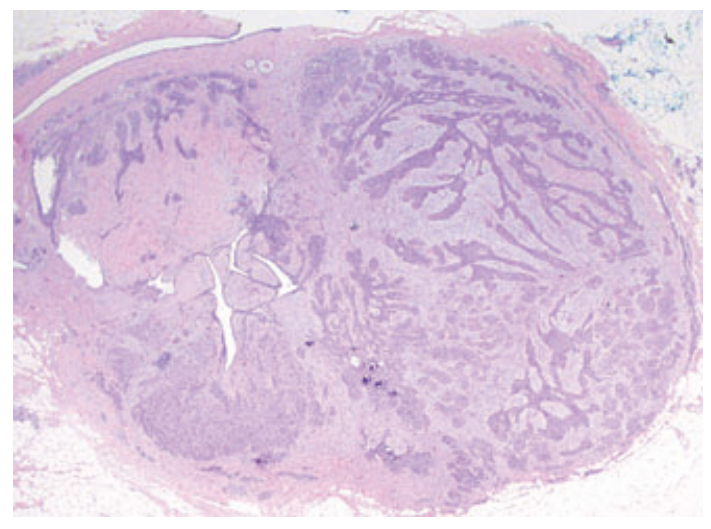

Figure 3. Invasive lobular carcinoma, alveolar variant, and lobular carcinoma in situ confined to a fibroadenoma (FA).

defined by lack of a myoepithelial layer as seen with all invasive breast cancers. It is more frequently identified as a minor component of otherwise classic ILC, but can also be seen in an isolated, "pure" form and it has been suggested that it may represent transition between LCIS and classic ILC. The prognostic significance of the alveolar variant is not certain, although recent literature suggests that it may be of better prognosis when compared to other ILC subtypes.
Invasive carcinoma limited to a FA is known to impart a more favorable prognosis. Irregular borders and pleomorphic and linear calcifications on mammogram may raise suspicion for malignancy, but unfortunately FAs harboring carcinoma cannot often be reliably distinguished from benign FAs via routine imaging. Neoplasia reported within FAs includes in situ and invasive ductal and lobular carcinomas; however, in situ carcinoma is more common and overall LCIS comprises the majority of neoplasia observed within FAs, a finding possibly explained by the theory that FAs derive from lobular origin. Improved prognosis and tendency toward in situ disease are thought to be a consequence of earlier discovery rather than intrinsic biologic differences.

This was an unusual presentation of breast carcinoma because the invasive component was purely of the uncommon alveolar variant of ILC and the tumor was completely confined to a FA. As often the case, this neoplasm could not be clinically distinguished from a benign FA, but was identified early (pT1bN0) which should impart a favorable overall prognosis. This case offers further evidence to support that older women presenting with a mass consistent with FA may warrant biopsy to rule out rare occult carcinoma. 